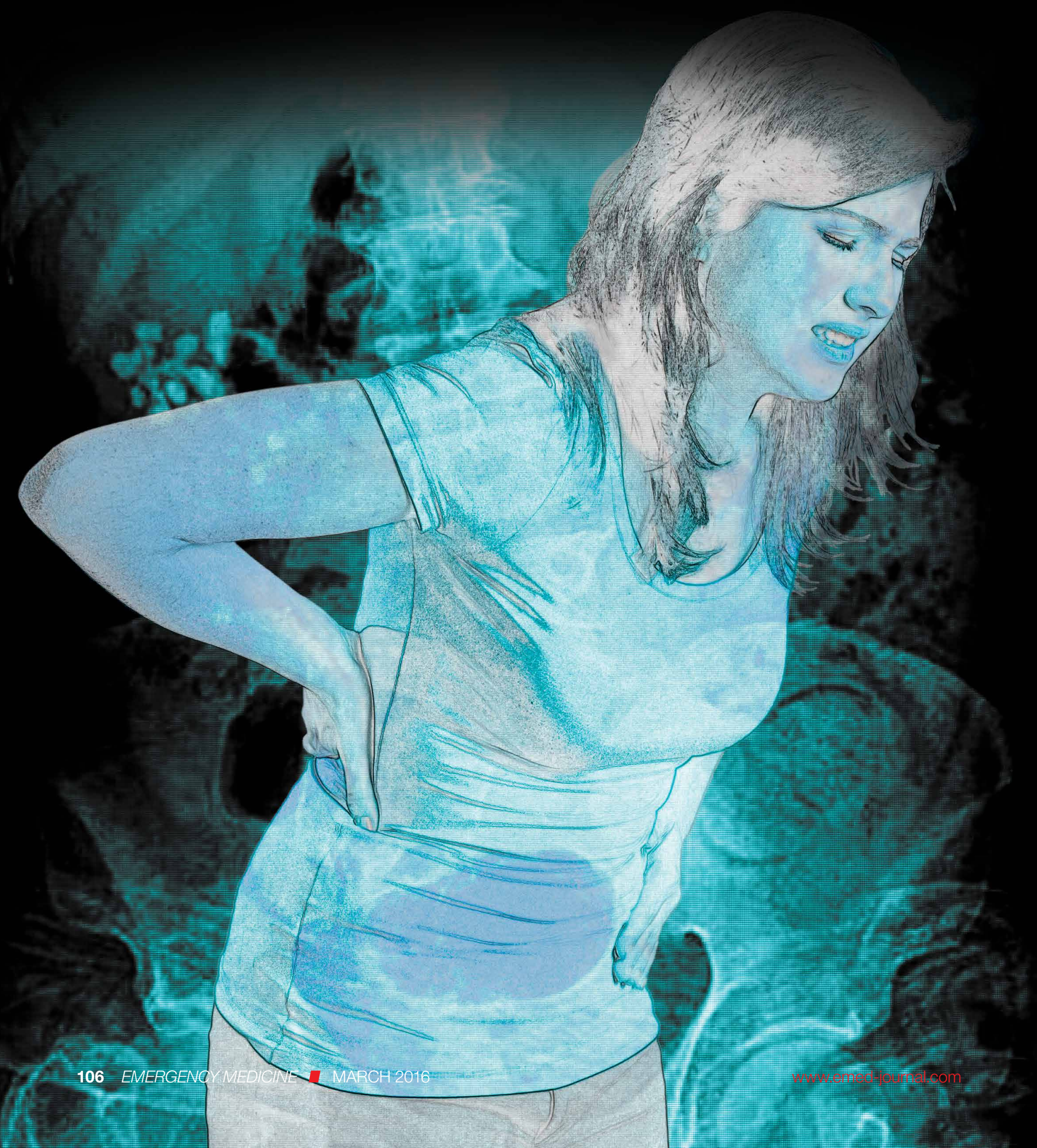




\section{Current Management of Nephrolithiasis}

Michael D. Parsa, MD; Michael M. Khafi, DO

\section{As the number of patients who present with signs and symptoms consistent with nephrolithiasis increases, the appropriate diag- nostic workup and management options continue to be debated.}

\section{Case}

A 39-year-old woman presented to the ED with a chief complaint of intermittent right flank pain that radiated into her groin area. She stated the pain had begun suddenly, 4 hours prior to arrival, and was accompanied by nausea and vomiting. The patient said that she had taken acetaminophen for the pain, but had received no relief. Regarding history, according to the patient, her last menstrual period ended 2 days earlier. She denied any urinary symptoms, diarrhea, or constipation. She had no history of abdominal surgery and was currently not on any medications.

The patient's vital signs at presentation were: temperature $98.7^{\circ} \mathrm{F}$; blood pressure, 130/90 mm Hg; heart rate, 110 beats/minute; and respiratory rate, 18 breaths/minute. Oxygen saturation was $98 \%$ on room air. On physical examination, she appeared to be in mild distress, pacing around the room. There was moderate right costovertebral tenderness on percussion; the abdomen was soft and nontender.

\section{Incidence}

As ED visits for nephrolithiasis are increasing, so too are the health-care costs asso- ciated with this condition. Between 1992 and 2009, emergent-care presentations for nephrolithiasis rose from 178 to 340 visits per 100,000 individuals. ${ }^{1}$ Approximately 1 in 11 people in the United States will be affected by nephrolithiasis during their lifetime. ${ }^{2}$ Estimated health-care costs associated with these complaints were roughly $\$ 2$ billion in 2000-an increase of 50\% since 1994. ${ }^{2}$

\section{Evaluation and Diagnosis \\ Laboratory Evaluation}

Urinalysis is one of the initial studies for patients with suspected nephrolithiasis. Although hematuria is a classic finding associated with renal calculi, its sensitivity on microscopic analysis is around $84 \%$. Therefore, the absence of hematuria does not exclude renal colic in the differential diagnosis. ${ }^{3}$

In addition to detecting hematuria, urinalysis can also reveal an underlying infection. One study by Abrahamian et $\mathrm{al}^{4}$ found that roughly $8 \%$ of patients presenting with acute nephrolithiasis had a urinary tract infection (UTI) - many without any clinical findings of infection. The presence of pyuria, however, has only 


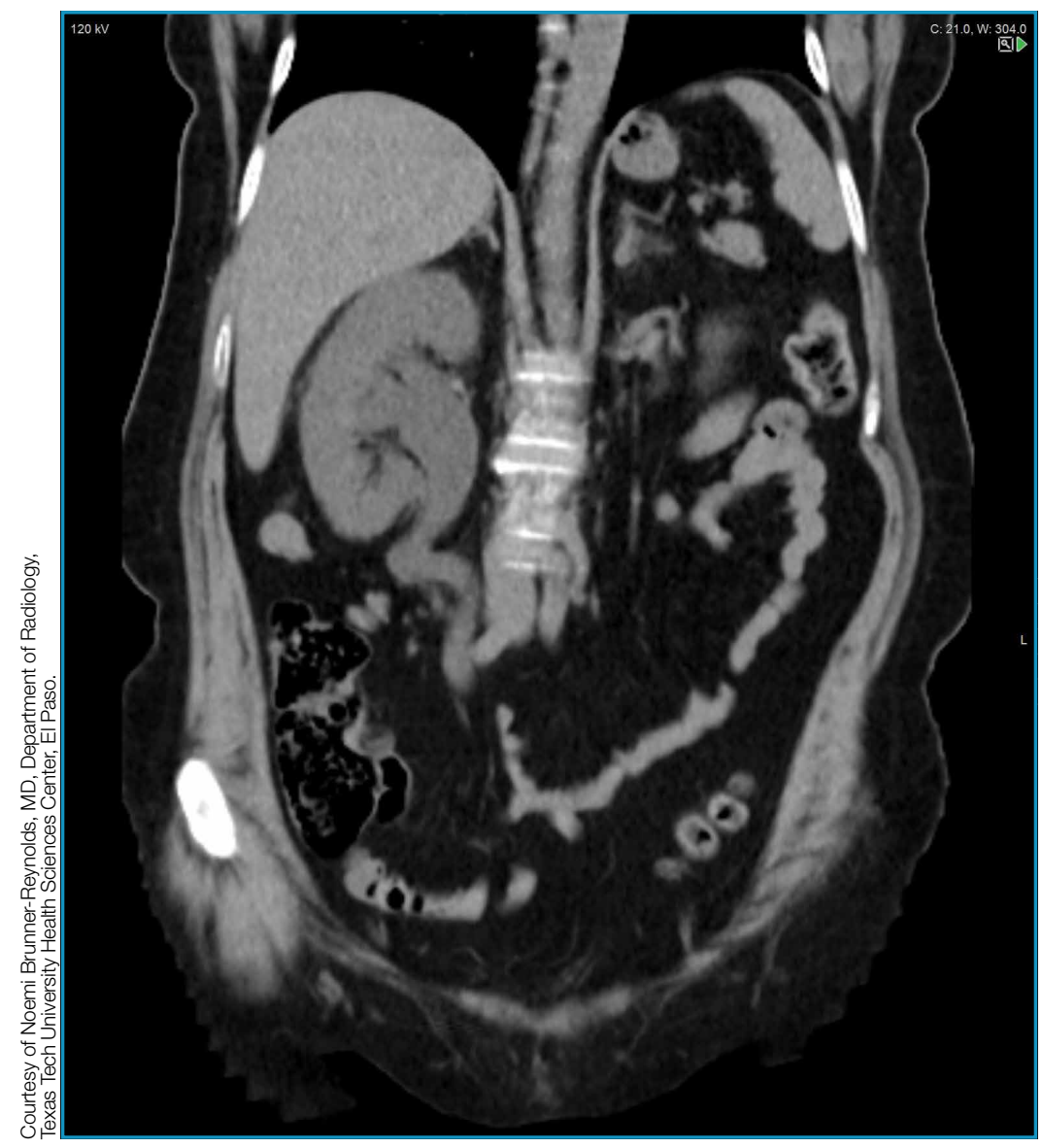

Figure 1. A noncontrast computed tomography image of the abdomen/pelvis demonstrating right-sided hydronephrosis secondary to renal calculus.

moderate accuracy in identifying UTIs in patients with kidney stones. ${ }^{4}$ If an infected stone cannot be excluded clinically, computed tomography (CT) is indicated.

Mild leukocytosis (ie, $<15,000$ cells/mcL) is another common finding in patients with acute renal colic. ${ }^{5} \mathrm{~A}$ leukocyte count $>15,000$ cells/mcL is suspicious for infection or other pathology. A blood-chemistry panel to evaluate renal function is appropriate as a baseline-particularly for patients in whom treatment with a nonsteroidal anti-inflammatory (NSAID) drug is anticipated.

\section{Unenhanced Computed Tomography}

With the ability to visualize renal calculi (Figure 1), the use of noncontrast CT has become a standard initial imaging modality in assessing patients with renal colic. Between 1992 and 2009, the use of CT to evaluate patients presenting with flank pain for suspected renal colic more than tripled from $21 \%$ to $71 \% .{ }^{6}$ An analysis performed by the American College of National Radiology Data Registry ${ }^{7}$ shows the mean radiation dose given by institutions for renal colic CT is unnecessarily high, and that few institutions follow CT-stone protocols aimed at minimizing radiation exposure while still maintaining proper diagnostic accuracy. A typical CT of the abdomen and pelvis is equivalent to over 100 two-view chest X-rays. ${ }^{8}$ Though controversial, data from a white paper by the American College of Radiology suggest that the ionizing radiation exposure from just one CT for renal colic causes an increase in lifetime cancer risk. ${ }^{9}$

Despite the increase in CT imaging to evaluate patients presenting to the ED with nephrolithiasis/flank pain, the proportion of patients diagnosed with a kidney stone remained the same between 2000 and 2008 , with no significant change in outcomes. ${ }^{10-12}$ Moreover, the use of CT as an initial imaging modality in patients presenting with flank pain-but with no sign of infection-is unlikely to reveal important alternative findings. ${ }^{13}$

Regarding the sensitivity of CT in detecting nephrolithiasis, one study demonstrates a sensitivity of $100 \%$ and a specificity of $94 \%$ for noncontrast CT. ${ }^{14}$ Controversy, however, still exists regarding the necessity and utility of CT in diagnosing nephrolithiasis, ${ }^{15}$ and CT is one of the top 10 tests included in the American College of Emergency Physicians (ACEP) 2014 Choosing Wisely campaign. In this campaign, ACEP recommended emergency physicians (EPs) avoid abdominal and pelvic CT in otherwise healthy patients younger than age 50 years who present with symptoms consistent with uncomplicated renal colic and who have a known history of nephrolithiasis or ureterolithia- 
sis. ${ }^{15}$ The ACEP also noted that CTs in this context do not often change treatment decisions and are associated with unnecessary radiation exposure and cost..$^{15}$

While keeping the aforementioned recommendations in mind, if an EP intends to refer a renal colic patient to a urologist a CT scan is necessary either in the ED or as an outpatient. In all cases (except perhaps in patients in whom there is a history of renal stones), the urologist will need this study to determine the size and location of the stone in order to provide recommendations for management.

\section{Ultrasound}

Ultrasound is another imaging modality with a high sensitivity in detecting renal calculi (Figure 2), and its role in the diagnosis of nephrolithiasis continues to evolve. A study by Smith-Bindman et $\mathrm{al}^{16}$ showed that in ED patients with symptoms suggestive of kidney stones, ultrasound as the initial imaging study was associated with lower cumulative radiation exposure than CT alone, with no significant differences in high-risk diagnosis with complications, serious adverse events, pain scores, return ED visits, or hospitalizations. In this study, $40 \%$ of patients who received point-ofcare (POC) ultrasound and $27 \%$ who received radiology ultrasound subsequently underwent CT in the ED. However, SmithBindman et al ${ }^{16}$ concluded that it is acceptable for EPs to consider replacing CT with ultrasound as the initial imaging study in most patients. It should be noted that this study excluded certain high-risk populations, including solitary kidney and renal transplant patients. In addition, patients with a high body mass index were also excluded from the study due to the unreliability of ultrasound in obese patients.

\section{Clinical Decision Score}

Moore et $\mathrm{al},{ }^{17}$ authors of the Size, Topography, Location, Obstruction, Number of stones, and Evaluation (STONE) scoring system, developed a classification system

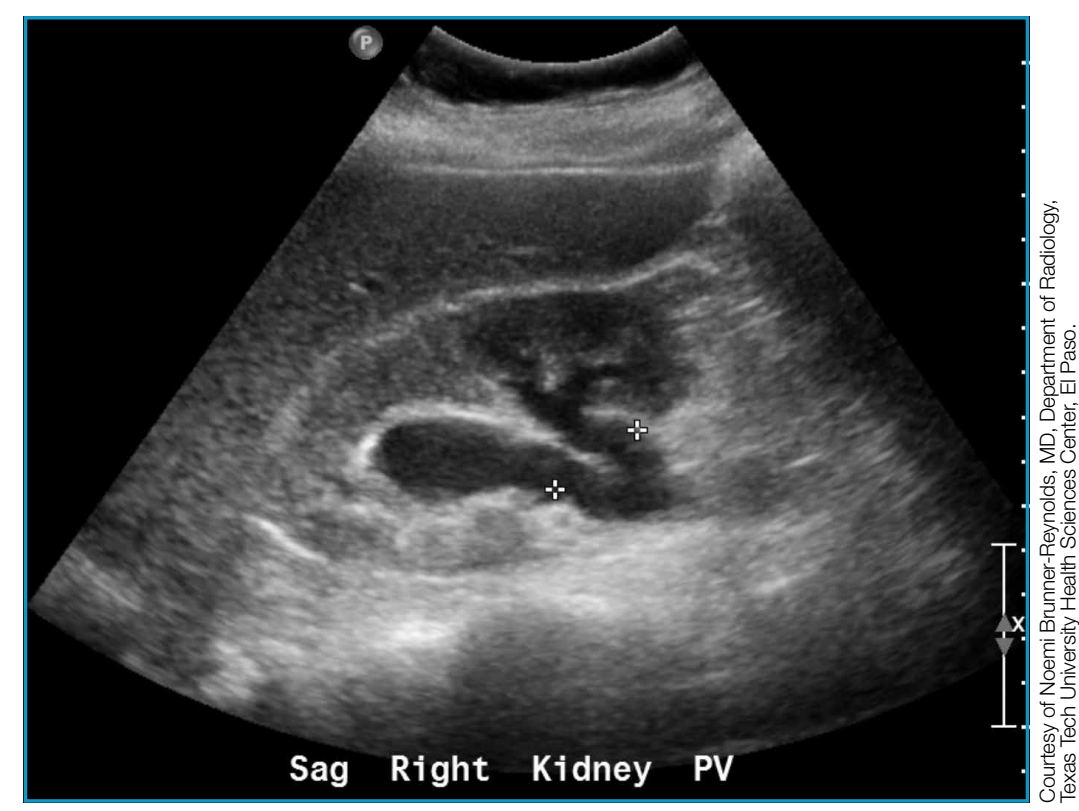

Figure 2. A sagittal right renal ultrasound image demonstrating hydronephrosis secondary to renal calculus.

for patients with suspected nephrolithiasis. This system places patients into low-, moderate-, and high-score groups, with corresponding probabilities of ureteral stone based on symptoms and epidemiological classifications.

The intent of the STONE system is to accurately predict, based on classification, the likelihood of a patient having a simple ureteral stone versus a more significant, complicated stone and to help guide which, if any, imaging studies are indicated. For example, a lower STONE score would help guide the decision to defer advanced imaging studies that would be unlikely to reveal an alternate serious diagnosis. Likewise, an individual with a high STONE score could potentially receive ultrasonography, reduced-dose CT, or no further imaging.

The STONE score performs fairly well and appears to be superior to physician gestalt, with an area under the receiver operating characteristic curve (AUC) of .78 compared to .68 with physician gestalt. This system, however, is not always accurate in its classification and has been 
shown to have $87 \%$ specificity at the high end to rule in stone and $96 \%$ sensitivity rate at the low end to rule out a stone. Of course, when using a clinical decision rule to rule in or rule out a stone, a tool with a very high specificity is preferred. Although the STONE scoring system does show promise, further studies are needed before it can be applied clinically. ${ }^{17}$

\section{Treatment}

\section{Analgesia}

By inhibiting prostaglandin synthesis, NSAIDs reduce inflammation and ureteral muscular hyperactivity. ${ }^{18} \mathrm{~A}$ recent Cochrane review of over 50 studies concluded that NSAIDs were effective in relieving acute renal colic pain..$^{19} \mathrm{~A}$ systematic review by Holdgate and Pollock ${ }^{20}$ shows that patients treated with NSAIDs achieve greater reductions in pain scores and are less likely to require additional analgesia in the short term compared to patients treated with opioids. Although opioid medications are effective in relieving pain associated with nephrolithiasis, this class of drugs can exacerbate the nausea often associated with this condition. This same study also showed that patients who were prescribed NSAIDs following an ED visit for renal colic required less medication for pain control, experienced less nausea, and had greater improvements in their pain. ${ }^{20}$

Nevertheless, the utility of opiates as an adjunct therapy should not be overlooked. For example, in patients with renal colic, numerous studies show treatment with a combination of an NSAID and opiate provides superior pain relief compared to either treatment modality in isolation. ${ }^{21} \mathrm{Opi}$ oid analgesia may be indicated in patients in whom NSAIDs are not recommended or contraindicated (eg, elderly patients, patients with renal disease). While NSAIDs address the underlying pathophysiology associated with renal colic, they are sometimes not the best treatment option. Depending on the situation, treatment with an opioid should instead be considered.

\section{Intravenous Fluid Therapy}

A 2012 Cochrane Review of randomized control trials (RCT) on intravenous (IV) fluid therapy hydration/diuretic use concluded that there was "no reliable evidence in the literature to support the use of diuretics and high-volume fluid therapy for people with acute ureteric colic." The review, however, did note that further investigation is warranted for a definitive answer. ${ }^{22}$ Another study by Springhart et $\mathrm{al}^{23}$ showed no difference in pain or stone expulsion between large-volume (2 L IV fluids over 2 hours) and small-volume fluid administration $(20 \mathrm{~mL} / \mathrm{h})$. Regarding administration, the use of IV fluids in renal colic is no different than the usual indications for fluid therapy in the ED and should be restricted to patients with signs of dehydration or kidney injury.

Many patients with renal colic will have decreased oral intake from the pain and nausea associated with the stone and may be vomiting. Under these circumstances, it is reasonable to rehydrate the patients, even though large-volume hydration with the intent of aiding stone expulsion or improving pain has not been shown efficacious. Conversely, in addition to the perceived benefit of rehydrating patients, a small amount of fluid hydration may improve the visualization of hydronephrosis on ultrasound..$^{24}$

\section{Medical Expulsive Therapy}

For many years, clinicians have considered the use of tamsulosin, an $\alpha 1$-receptor blocker, as well as nifedipine, a calcium channel blocker, in treating renal colic due to the theoretical benefit of reducing ureteral smooth muscle spasm/constriction thus expediting stone passage. Over the years, dozens of studies showed positive benefit in the use of medical expulsive therapy (MET). A 2014 Cochrane Review demonstrated that patients treated with $\alpha 1$ blockers experienced a higher stone-free rate and shorter time to stone expulsion, and concluded that $\alpha 1$-blockers should be 
offered as one of the primary treatment modalities in MET. ${ }^{25}$ This review, however, has been criticized for using a number of studies with very small patient samples, non-peer-reviewed abstracts, and lowquality study designs. ${ }^{26}$

More recently, in April 2015, Lancet published a large RCT from 24 hospitals in the United Kingdom, comparing placebo versus $400 \mathrm{mcg}$ tamsulosin and $30 \mathrm{mg}$ nifedipine. The authors concluded that "tamsulosin $400 \mathrm{mcg}$ and nifedipine $30 \mathrm{mg}$ are not effective at decreasing the need for further treatment to achieve stone clearance in 4 weeks for patients with expectantly managed ureteric colic." ${ }^{27}$ Another large double-blind, placebo-controlled, randomized, multicenter trial by Furyk et $\mathrm{al}^{28}$ in July 2015 went a step further and evaluated distal stones, which have historically caused complications requiring intervention. They concluded that there was "no benefit overall of $0.4 \mathrm{mg}$ of tamsulosin daily for patients with distal ureteric calculi less than or equal to $10 \mathrm{~mm}$ in terms of spontaneous passage, time to stone passage, pain, or analgesia requirements. In the subgroup with large stones (5 to $10 \mathrm{~mm}$ ), tamsulosin did increase passage and should be considered." ${ }^{28}$ Based on these recent studies, the use of tamsulosin in patients with stones larger than $5 \mathrm{~mm}$-but not those with smaller stones-appears to be an appropriate treatment option.

\section{Patient Disposition}

The American Urological Association cited indications for urgent/emergent urological interventions necessitating the need for inpatient admission and further workup. ${ }^{29}$ Patients who do not fall into any of the categories outlined in the Table may be seen on an outpatient basis. These patients may be treated symptomatically until they can follow up with a urologist, who will determine expectant management versus intervention.

In many communities, initial followup with a primary care physician (PCP),
Table. Indications for Urgent Urology Consultation With Urinary Stones ${ }^{29}$

Oral Intolerance

Pain Refractory to Analgesics

Impending Renal Deterioration

Obstructed Upper Tract with Infection

Patient Preference

Reprinted with permission from the American Urological Association.

rather than a urologist, is standard for patients who are likely to pass the stone spontaneously-specifically those with nonobstructing stones $<5 \mathrm{~mm}$ in diameter and no history of prior complicated kidney stone. Any patient discharged home with a suspected or confirmed diagnosis of nephrolithiasis should be instructed to return to the ED if he or she is unable to take the prescribed medications due to excessive nausea/vomiting; becomes febrile; develops severe pain despite oral medication; or develops any other worrisome symptoms. All of these indicate that he or she may have progressed to complicated nephrolithiasis requiring further workup and potential intervention (Table). Computed tomography should be pursued in a patient whose stone is symptomatic enough to warrant inpatient admission. For example, a patient who is febrile or whose urinalysis is suggestive of infection-in addition to a high clinical suspicion of renal colic-should undergo CT evaluation to rule out an obstructing infected stone or another possible diagnosis. Computed tomography investigation is required in any patient who presents with colicky pain or flank pain and whose condition is considered complicated.

\section{Prognosis}

The majority of stones $<5 \mathrm{~mm}$ will pass spontaneously. ${ }^{30}$ Larger stones may still pass spontaneously but are more likely 
to require lithotripsy or other urologic intervention; therefore, patients with stones $>5 \mathrm{~mm}$ should be referred to urology services. ${ }^{30}$

\section{Recurrence}

Patients with a first-time kidney stone have a $30 \%$ to $50 \%$ chance of disease recurrence within 5 years, ${ }^{31}$ and a $60 \%$ to $80 \%$ chance of recurrence during their lifetime. ${ }^{32}$ Those with a family history of nephrolithiasis are likely to develop an earlier onset of stones as well as experience more frequent recurrent episodes. ${ }^{33}$ Patients with recurrent disease should undergo outpatient risk stratification, including stone-composition analysis and assessment for modifiable risk factors.

\section{Case Conclusion}

The patient's urinalysis demonstrated microscopic hematuria; blood urea nitrogen and creatinine levels were within normal limits. As the patient was tachycardic and appeared mildly dehydrated, an IV infusion of $1 \mathrm{~L}$ normal saline was initiated, along with ketorolac and ondansetron for

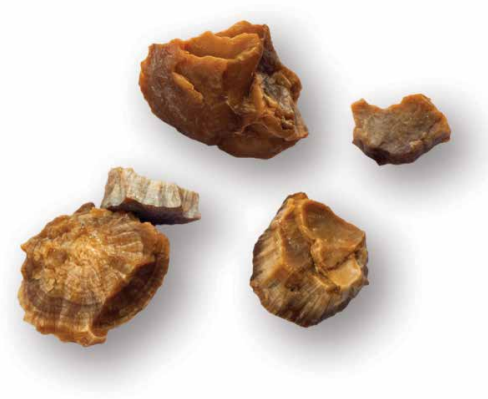
symptomatic relief. A POC ultrasound of the right kidney revealed mild-to-moderate hydronephrosis; the left kidney appeared sonographically normal. Since this patient had no history of nephrolithiasis, a nonenhanced CT of the abdomen was obtained, which revealed moderate, right-sided hydronephrosis and a 3-mm distal ureteral stone. Once the patient's symptoms were controlled, she was discharged home with a prescription for ibuprofen for symptomatic relief and instructions to follow up with her PCP.

\section{Conclusion}

The evaluation and treatment of nephrolithiasis is important due to its increasing prevalence, as well as implications on costs to the health-care system and to patients themselves. The workup and treatment of nephrolithiasis has been and continues to be the subject of much controversy. Until very recently, treatment recommendations were founded on physiological theories more so than robust research. In an era where improved imaging technology is becoming more readily available in the ED, EPs should weigh the pros and cons of its utilization for common ED complaints such as nephrolithiasis.

\section{References}

1. Scales CD Jr, Smith AC, Hanley JM, Saigal CS; Urologic Diseases in America Project. Prevalence of kidney stones in the United States. Eur Urol. 2012;62(1):160-165.

2. Pearle MS, Calhoun EA, Curhan GC; Urologic Diseases of America Project: urolithiasis. J Urol. 2005;173(3):848-857.

3. Luchs JS, Katz DS, Lane MJ et al. Utility of hematuria testing in patients with suspected renal colic: correlation with unenhanced helical CT results. Urology. 2002;59(6):839-842.

4. Abrahamian FM, Krishnadasan A, Mower WR, Moran GJ, Talan DA. Association of pyuria and clinical characteristics with the presence of urinary tract infection among patients with acute nephrolithiasis. Ann Emerg Med. 2013;62(5):526-533.

5. Yilmaz S, Pekdemir M, Aksu NM, Koyuncu N, Cinar O, Akpinar E. A multicenter case-control study of diagnostic tests for urinary tract infection in the presence of urolithiasis. Urol Res. 2011;40(1):61-65. doi:10.1007/s00240-011-0402-x.

6. Fwu CW, Eggers PW, Kimmel PL, Kusek JW, Kirkali Z. Emergency department visits, use of imaging, and drugs for urolithiasis have increased in the United States. Kidney Int. 2013;83(3):479-486. doi:10.1038/ ki.2012.419.

7. Lukasiewicz A, Bhargavan-Chatfield M, Coombs L, et al. Radiation dose index of renal colic protocol CT studies in the United States: a report from the American College of Radiology National Radiology Data Registry. Radiology. 2014;271(2):445-451. doi:10.1148/radiol.14131601.

8. Mettler FA Jr, Huda W, Yoshizumi TT, Mahesh M. Effective doses in radiology and diagnostic nuclear medicine: a catalog. Radiology. 248(1):254-263.

9. Amis ES Jr, Butler PF, Applegate KE, et al; American College of Radiology. American College of Radiology white paper on radiation dose in medicine. $J \mathrm{Am}$ Coll Radiol. 2007;4(5):272-284.

10. Hyams ES, Korley FK, Pham JC, Matlaga BR. Trends in imaging use during the emergency department evaluation of flank pain. J Urol. 2011;186(6):22702274. doi:10.1016/j.juro.2011.07.079.

11. Ripollés T, Agramunt M, Errando J, Martínez MJ, Coronel B, Morales M. Suspected ureteral colic: plain film and sonography vs unenhanced helical CT. A prospective study in 66 patients. Eur Radiol. 2004;14(1):12936. doi:10.1007/s00330-003-1924-1926.

12. Westphalen AC, Hsia RY, Maselli JH, Wang R, Gonzales R. Radiological imaging of patients with suspected urinary tract stones: national trends, diagnoses, and predictors. Acad Emerg Med. 2011;18(7):699707. doi:10.1111/j.1553-2712.2011.01103.x.

13. Moore CL, Daniels B, Singh D, Luty S, Molinaro A. Prevalence and clinical importance of alternative 
causes of symptoms using a renal colic computed tomography protocol in patients with flank or back pain and absence of pyuria. Acad Emerg Med. 2013;20(5):470-478. doi:10.1111/acem.12127.

14. Chen MY, Zagoria RJ. Can noncontrast helical computed tomography replace intravenous urography for evaluation of patients with acute urinary tract colic? J Emerg Med. 1999;17(2):299-303.

15. American College of Emergency Physicians. Five things physicians and patients should question. Choosing Wisely Web site. 2013;10:1-5. Available at: http://www.choosingwisely.org/societies/americancollege-of-emergency-physicians/. Accessed February 10, 2016

16. Smith-Bindman R, Aubin C, Bailitz J, et al. Ultrasonography versus computed tomography for suspected nephrolithiasis. N Engl J Med. 2014;371(12):11001110. doi:10.1056/nejmoa1404446.

17. Moore CL, Bomann S, Daniels B, et al. Derivation and validation of a clinical prediction rule for uncomplicated ureteral stone-the STONE score: retrospective and prospective observational cohort studies. BMJ. 2014;348:g2191. doi:10.1136/bmj. g2191.

18. Cole RS, Fry CH, Shuttleworth KE. The action of the prostaglandins on isolated human ureteric smooth muscle. Br J Urol. 1988;61(1):19-26.

19. Afshar K, Jafari S, Marks AJ, Eftekhari R, McNeily AE. Nonsteroidal anti-inflammatory drugs (NSAIDs) and non-opioids for acute renal colic. Cochrane Database Syst Rev. 2015;6:CD006027. doi:10.1002/14651858.CD006027.pub2.

20. Holdgate A, Pollock T. Systematic review of the relative efficacy of non-steroidal anti-inflammatory drugs and opioids in the treatment of acute renal colic. BMJ. 2004;328(7453):1401. doi:10.1136/ bmj.38119.581991.55.

21. Safdar B, Degutis LC, Landry K, Vedere SR, Moscovitz HC, D’Onofrio G. Intravenous morphine plus ketorolac is superior to either drug alone for treatment of acute renal colic. Ann Emerg Med. 2006;48(2):173-181, 181.e1. doi:10.1016/j.annemergmed.2006.03.013.

22. Worster AS, Bhanich Supapol W. Fluids and diuretics for acute ureteric colic. Cochrane Database Syst Rev. 2012;15;2:CD004926. doi:10.1002/14651858. CD004926.pub3
23. Springhart WP, Marguet CG, Sur RL, et al. Forced versus minimal intravenous hydration in the management of acute renal colic: a randomized trial. J. Endourol. 2006;20(10):713-716. doi:10.1089/ end.2006.20.713.

24. Morse JW, Hill R, Greissinger WP, Patterson JW, Melanson SW, Heller MB. Rapid oral hydration results in hydronephrosis as demonstrated by bedside ultrasound. Ann Emerg Med. 1999;34(2):134-140. doi:10.1016/s0196-0644(99)70221-0.

25. Campschroer T, Zhu Y, Duijvesz D, Grobbee DE, Lock MT. Alpha-blockers as medical expulsive therapy for ureteral stones. Cochrane Database Syst Rev. 2014;4:CD008509. doi:10.1002/14651858. CD008509.pub2.

26. Radecki R. Sadly inadequate Cochrane review of renal colic. Emergency Medicine Literature of Note. 2014. Available at: http://www.emlitofnote. com/2014/04/sadly-inadequate-cochrane-review-of html. Accessed February 10, 2016.

27. Pickard R, Starr K, MacLennan G, et al. Medical expulsive therapy in adults with ureteric colic: a multicentre, randomised, placebo-controlled trial. Lancet. 2015;386(9991):341-349. doi:10.1016/S01406736(15)60933-3.

28. Furyk JS, Chu K, Banks C, et al. Distal ureteric stones and tamsulosin: a double-blind, placebo-controlled, randomized, multicenter trial. Ann Emerg Med. 2016;67(1):86-95.e2. doi:10.1016/j.annemergmed.2015.06.001.

29. Kidney stones. American Urological Association Web site. 2016. Available at: https://www.auanet.org/education/kidney-stones.cfm. Accessed February 10, 2016

30. Miller OF, Kane CJ. Time to stone passage for observed ureteral calculi: a guide for patient education. J Urol. 1999;162(3 Pt 1):688-690.

31. Lipkin ME, Preminger GM. Demystifying the medical management of nephrolithiasis. Rev Urol. 2011;13(1):34-38

32. Morton AR, Iliescu EA, Wilson JW. Nephrology: 1. Investigation and treatment of recurrent kidney stones. CMAJ. 2002;166(2):213-218.

33. Koyuncu HH, Yencilek F, Eryildirim B, Sarica K. Family history in stone disease: how important is it for the onset of the disease and the incidence of recurrence? Urol Res. 2010;38(2):105-109. doi:10.1007/ s00240-009-0249-6. 